\title{
Glioma: Application of Histogram Analysis of Pharmacokinetic Parameters from T1-Weighted Dynamic Contrast-Enhanced MR Imaging to Tumor Grading
}

\author{
S.C. Jung, J.A. Yeom, J.-H. Kim, I. Ryoo, S.C. Kim, H. Shin, A.L. Lee, T.J. Yun, C.-K. Park, C.-H. Sohn, S.-H. Park, and S.H. Choi
}

\begin{abstract}
BACKGROUND AND PURPOSE: The usefulness of pharmacokinetic parameters for glioma grading has been reported based on the perfusion data from parts of entire-tumor volumes. However, the perfusion values may not reflect the entire-tumor characteristics. Our aim was to investigate the feasibility of glioma grading by using histogram analyses of pharmacokinetic parameters including the volume transfer constant, extravascular extracellular space volume per unit volume of tissue, and blood plasma volume per unit volume of tissue from T1-weighted dynamic contrast-enhanced perfusion MR imaging.
\end{abstract}

MATERIALS AND METHODS: Twenty-eight patients (14 men, 14 women; mean age, 49.75 years; age range, 25-72 years) with histopathologically confirmed gliomas (World Health Organization grade II, $n=7$; grade III, $n=8$; grade IV, $n=13$ ) were examined before surgery or biopsy with conventional MR imaging and T1-weighted dynamic contrast-enhanced perfusion MR imaging at 3T. Volume transfer constant, extravascular extracellular space volume per unit volume of tissue, and blood plasma volume per unit volume of tissue were calculated from the entire-tumor volume. Histogram analyses from these parameters were correlated with glioma grades. The parameters with the best percentile from cumulative histograms were identified by analysis of the area under the curve of the receiver operating characteristic analysis and were compared by using multivariable stepwise logistic regression analysis for distinguishing high-from low-grade gliomas.

RESULTS: All parametric values increased with increasing glioma grade. There were significant differences among the 3 grades in all parameters $(P<.01)$. For the differentiation of high- and low-grade gliomas, the highest area under the curve values were found at the 98 th percentile of the volume transfer constant (area under the curve, 0.912 ; cutoff value, 0.277 ), the 90th percentile of extravascular extracellular space volume per unit volume of tissue (area under the curve, 0.939; cutoff value, 19.70), and the 84th percentile of blood plasma volume per unit volume of tissue (area under the curve, 0.769 ; cutoff value, 11.71). The 98th percentile volume transfer constant value was the only variable that could be used to independently differentiate high- and low-grade gliomas in multivariable stepwise logistic regression analysis.

CONCLUSIONS: Histogram analysis of pharmacokinetic parameters from whole-tumor volume data can be a useful method for glioma grading. The 98th percentile value of the volume transfer constant was the most significant measure.

ABBREVIATIONS: $A U C=$ area under the curve; $D C E=$ dynamic contrast-enhanced; $K^{\text {trans }}=$ volume transfer constant; $R O C=$ receiver operating characteristic analysis; $\mathrm{Ve}=$ extravascular extracellular space volume per unit volume of tissue; $\mathrm{Vp}=$ blood plasma volume per unit volume of tissue

T he growth of tumors beyond a certain size depends on the development of a vascular supply that meets the metabolic requirements of the neoplastic tissue. ${ }^{1-4}$ Newly developed vascu-

Received July 11, 2013; accepted after revision October 21.

From the Departments of Radiology (S.C., J.a.Y., J.-H.K., I.R., S.C.K., H.S., A.L.L., T.J.Y., C.-H.S., S.H.C.), Neurosurgery (C.-K.P.), and Pathology (S.-H.P.), Seoul National University College of Medicine, Seoul, Republic of Korea; Center for Nanoparticle Research (S.H.C.), Institute for Basic Science, and School of Chemical and Biological Engineering, Seoul National University, Seoul, Republic of Korea.

This study was supported by a grant from the National R\&D Program for Cancer Control, Ministry of Health and Welfare, Republic of Korea (1120300); the Korea Healthcare Technology R\&D Projects, Ministry for Health, Welfare and Family Affairs (A112028 and HI13C0015); and the Research Center Program of the Institute for Basic Science in Korea. lar networks of tumors show increased tortuosity, lack of maturity, and increased permeability to macromolecules due to the presence of large endothelial cell gaps. ${ }^{5,6}$ In particular, high-grade gliomas are associated with a higher proportion of immature and hyperpermeable vessels. ${ }^{7,8}$

Angiogenesis and the microvascular system of gliomas have recently been the focus of grading before treatment and for evaluating posttherapy status with perfusion MR imaging. With con-

\footnotetext{
Please address correspondence to Seung Hong Choi, MD, PhD, Department of Radiology, Seoul National University College of Medicine, 28, Yongon-dong, Chongno-gu, Seoul, 110-744, Republic of Korea; e-mail: verocay@snuh.org

- Indicates open access to non-subscribers at www.ajnr.org

http://dx.doi.org/10.3174/ajnr.A3825
} 
trast media, the dynamic change of signal intensity can be measured and converted into an analysis of perfusion changes. From the signal-time curves, many pharmacokinetic parameters can be extracted for the characterization of gliomas. Cerebral blood volume and cerebral blood flow, vascular permeability, and extravascular extracellular volume have been proposed as representative parameters derived from T1-weighted dynamic contrast-enhanced (DCE) perfusion-weighted imaging in the differentiation of glioma grades. ${ }^{8-16}$ More recently, many reports based on T1weighted DCE-PWI MR imaging have introduced volume transfer constant $K^{\text {trans }}$, extravascular extracellular space volume per unit volume of tissue ( $\mathrm{Ve}$ ), and blood plasma volume per unit volume of tissue $(\mathrm{Vp})$ as feasible parameters for glioma grading. ${ }^{8,10,17,18} K^{\text {trans }}$ and Ve are pharmacokinetic parameters wellknown for reflecting permeability. $K^{\text {trans }}$ is the volume transfer constant between the plasma and extravascular extracellular space. Ve is the extravascular extracellular space per unit volume of tissue and is known as leakage space. ${ }^{19,20} \mathrm{Vp}$ is defined as the blood plasma volume per unit volume of tissue, which may be a marker of angiogenic activity in a tumor. ${ }^{13}$ However, previous studies collected the perfusion data from parts of entire-tumor volumes. Therefore, the presented values may not have reflected the entire tumor characteristics.

In this study, we obtained the pharmacokinetic parameters from the entire-tumor volume by using T1-weighted DCE MR images. We hypothesized that a histogram-based analysis of the pharmacokinetic parameters from the entire-tumor volume would be helpful for glioma grading because high-grade gliomas have a wide spectrum of histologic features, ranging from grade II to IV; this wide spectrum may account for sampling biases. The purposes of this study were to investigate the feasibility of using a histogram analysis of pharmacokinetic parameters, including $K^{\text {trans }}, \mathrm{Ve}$, and $\mathrm{Vp}$, for glioma grading and to explore the diagnostic performance of the histogram analysis of these parameters.

\section{MATERIALS AND METHODS}

This retrospective study was approved by our institutional review board. Patient informed consent was waived.

\section{Patients}

Between January 2010 and October 2012, fifty patients with initial DCE MR imaging at our institution (Seoul National University Hospital) were diagnosed with astrocytic tumors on the basis of the World Health Organization classification for tumors of the central nervous system. This study included 28 patients with the following inclusion criteria: 1) MR imaging was performed with a 3T scanner before treatment or biopsy; and 2) grade II, III, and IV gliomas were diagnosed. There were 14 male and 14 female patients enrolled (mean age, 49.75 years; age range, 25-72 years). In the enrolled patients, there were 28 gliomas (grade II, 7 gliomas; grade III, 8 gliomas; grade IV, 13 gliomas). Grade II gliomas were classified as low-grade, and grades III and IV were classified as high-grade.

\section{MR Imaging Protocol}

The MR imaging protocol consisted of conventional MR imaging and DCE MR imaging by using a 3T scanner with a 32-channel head coil (Magnetom Verio; Siemens, Erlangen, Germany). Conventional MR imaging included the following: T1-weighted imaging, such as transverse spin-echo imaging, before and after contrast enhancement; multiplanar reconstructed transverse, coronal imaging with a sagittal 3D magnetization-prepared rapid acquisition of gradient echo sequence, before and after contrast enhancement; transverse fluid-attenuated inversion recovery; and transverse T2-weighted imaging with turbo spin-echo sequences. Contrast-enhanced T1WI was performed after the intravenous administration of gadobutrol (Gadovist; Bayer Schering Pharma, Berlin, Germany), at a dose of $0.1 \mathrm{mmol} / \mathrm{kg}$ of body weight. Transverse spin-echo T1WI was performed with the following parameters: TR, $558 \mathrm{~ms}$; TE, $9.8 \mathrm{~ms}$; flip angle, $70^{\circ}$; matrix, $384 \times$ 187; FOV, $175 \times 220 \mathrm{~mm}$; section thickness, $5 \mathrm{~mm}$; and NEX, 1 . We obtained the 3D-MPRAGE sequences by using the following parameters: TR, $1500 \mathrm{~ms}$; TE, $1.9 \mathrm{~ms}$; flip angle, $9^{\circ}$; matrix, $256 \times$ 232; FOV, $220 \times 250$; section thickness, $1 \mathrm{~mm}$; and NEX, 1 . The parameters in axial FLAIR imaging were the following: TR, 9000 ms; TE, $97 \mathrm{~ms}$; TI, $2500 \mathrm{~ms}$; flip angle, 130'; matrix, $384 \times 348$; FOV, $199 \times 220$; and section thickness, $5 \mathrm{~mm}$. The parameters of the transverse T2WI were as follows: TR, $5160 \mathrm{~ms}$; TE, $91 \mathrm{~ms}$; flip angle, $124^{\circ}-130^{\circ}$; matrix, $640 \times 510-580$; FOV , 175-199 $\times 220$; section thickness, $5 \mathrm{~mm}$; and NEX, 3 .

DCE MR imaging was performed with $3 \mathrm{D}$ gradient-echo T1WI after the intravenous administration of gadobutrol ( 0.1 $\mathrm{mmol} / \mathrm{kg}$ of body weight) at a rate of $4 \mathrm{~mL} / \mathrm{s}$ by using a power injector (Spectris MR injector; MedRad, Indianola, Pennsylvania). A $30-\mathrm{mL}$ bolus injection of saline followed at the same injection rate. For each section, 40 images were acquired at intervals equal to the TR. The parameters were as follows: TR, $2.8 \mathrm{~ms}$; TE, $1.0 \mathrm{~ms}$; flip angle, $10^{\circ}$; matrix, $192 \times 192$; section thickness, $3 \mathrm{~mm}$; FOV, $240 \times 240 \mathrm{~mm}$; voxel size, $1.25 \times$ $1.25 \times 3 \mathrm{~mm}^{3}$; pixel bandwidth, $789 \mathrm{~Hz}$; total acquisition time, 1 minute 30 seconds.

\section{Image Analysis}

DCE MR images were processed by using the MR perfusion analysis method (nordicICE; Nordic Neuro Lab, Bergen, Norway), in which FLAIR or T2WI was used for structural imaging. On the basis of the 2-compartment pharmacokinetic model proposed by Tofts and Kermode, ${ }^{21}$ we used the perfusion analysis method to calculate pharmacokinetic parameters, including $K^{\text {trans }}, \mathrm{Ve}$, and $\mathrm{Vp} .^{21}$ Deconvolution with the arterial input function was performed in the pharmacokinetic model. For each tumor, the arterial input function was determined in intracranial tumor-supplying arteries near the tumor. Perfusion analysis was able to generate an arterial input function curve and a concentrationtime curve of the tumor for each tumor-supplying artery in a region of interest. Two neuroradiologists (S.C.J. and S.H.C.) manually defined the ROIs by consensus. They chose an appropriate arterial input function curve to show the ideal relationship between the input function curve and the concentration-time curve. The arterial input function curves had higher values than the concentration-time curves and showed rapid wash-in and slow washout patterns, whereas the concentration-time curves showed a steady increase in the wash-in slope. The baseline T1 value was fixed at $1000 \mathrm{~ms}$ in this study. ${ }^{22}$ 


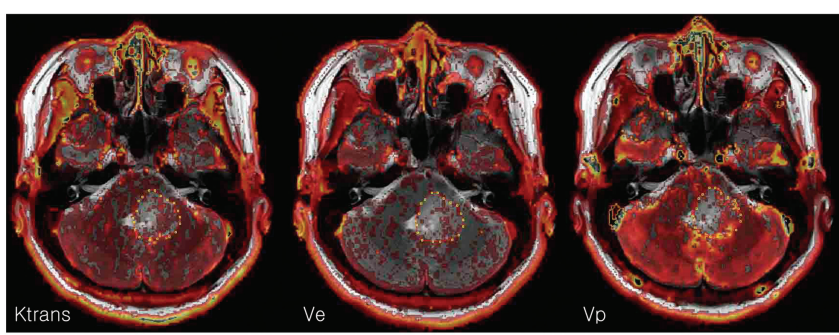

A

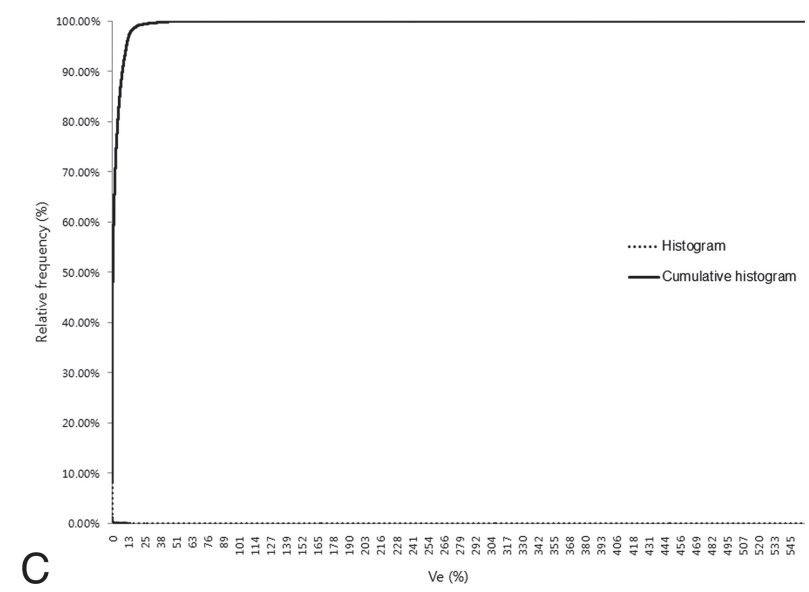

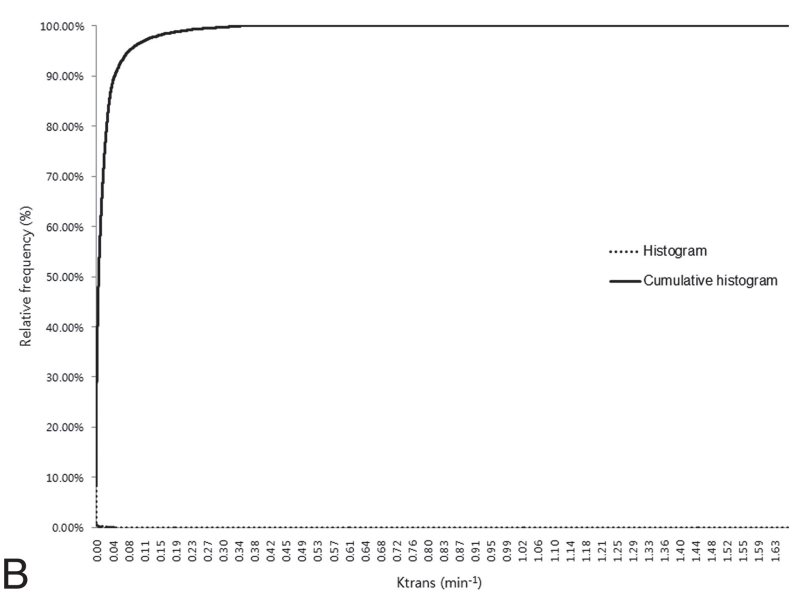

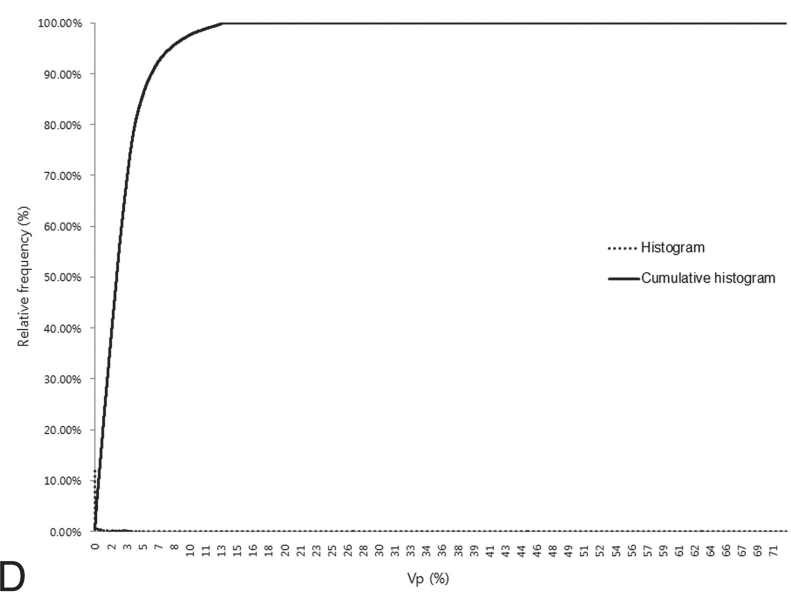

FIG 1. ROIs manually defined on axial T2WI coregistered with parametric maps in a 43-year-old woman with a grade II glioma ( $A$ ) and the histogram and cumulative histogram from the entire-tumor volume $(B-D)$ are presented.

Pharmacokinetic parameters, including $K^{\text {trans }}, \mathrm{Ve}$, and $\mathrm{Vp}$, were measured from each region of interest by a neuroradiologist (S.C.J.). The perfusion analysis method was used to calculate the pharmacokinetic parameters and to present these parameters as parametric maps. The coregistration between the structural images and parametric maps (color overlay) was accomplished automatically by using mutual information based on an algorithm that facilitated the search for an optimal rigid transformation aligning the 2 datasets. ${ }^{23-25}$ The 2 reviewers defined the margins of the tumors on each axial plane by consensus, manually outlining the margins on the T2WI or FLAIR image by using the perfusion analysis workstation. The outline of the tumor region of interest was defined excluding the cystic or necrotic regions and intralesional macrovessels (Figs $1 A, 2 A$, and $3 A$ ). On a pixel-by-pixel basis, all values of the parameters were calculated from an outline (region of interest) on an axial plane. The overall values for each tumor were obtained by summing the values from every plane. Finally, the total parametric values from the entire-tumor volume were acquired and recorded for each tumor.

\section{Statistical Analysis}

Commercially available software (MedCalc for Windows, Version 11.1.1.0; MedCalc Software, Mariakerke, Belgium) was used for the analysis. The Kolmogorov-Smirnov test was used to determine whether values were normally distributed. For all statistical analyses, a 2 -tailed $P$ value $<.05$ was considered indicative of a statistically significant difference.

The mean and SD of the parametric values were calculated for each parameter. The values were compared according to the grade by using the unpaired $t$ test.

The histograms were plotted with the parametric values on the $\mathrm{x}$-axis, with a bin size of $1 \times 10^{-4} \mathrm{~min}^{-1}$ for $K^{\text {trans }}, 1 \times 10^{-2}$ for $\mathrm{Ve}$, and $1 \times 10^{-2}$ for $\mathrm{Vp}$. The relative frequency was plotted on the $y$-axis of the histograms as a percentage of the total tumor volume, calculated by dividing the frequency in each bin by the total number of pixels. ${ }^{26}$ The ranges of the bins were chosen as $0-1.66$ in $K^{\text {trans }}, 0-5.5765$ in Ve, and $0-0.7201$ in Vp because $1.66,5.5765$, and 0.7201 were the highest values for each parameter, respectively. The cumulative histograms were built from the aforementioned histograms. The relative cumulative frequency on the $y$-axis was expressed as a percentage summating the relative frequency from the first bin to a specified bin, and the parametric values were plotted on the $\mathrm{x}$-axis (Figs $1 B-D, 2 B-D$, and $3 B-D)$.

A corresponding parametric value to a specific one from the 50th percentile (C50) to the 100th percentile (C100) was calcu- 


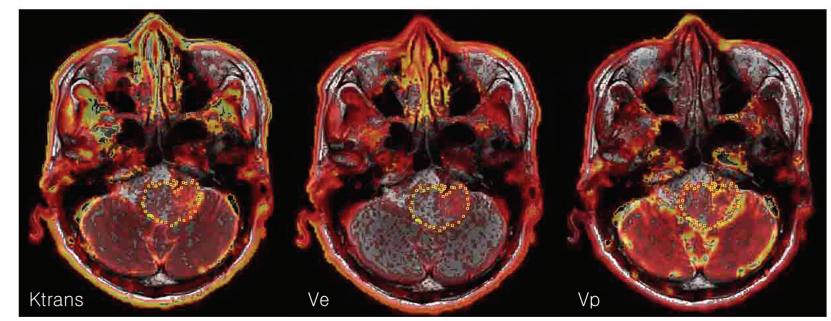

A

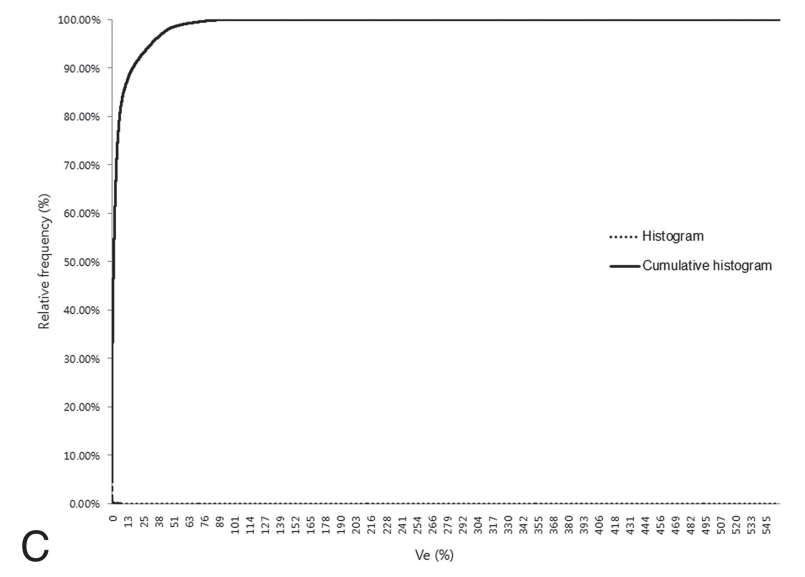

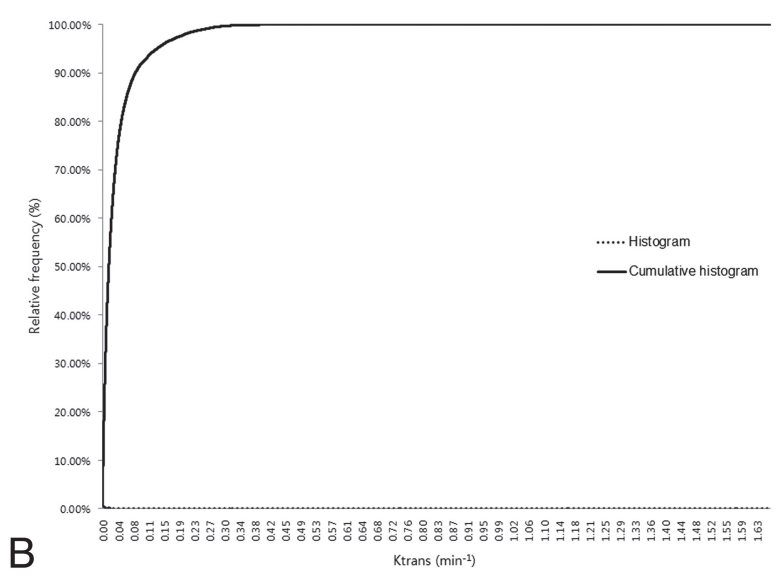

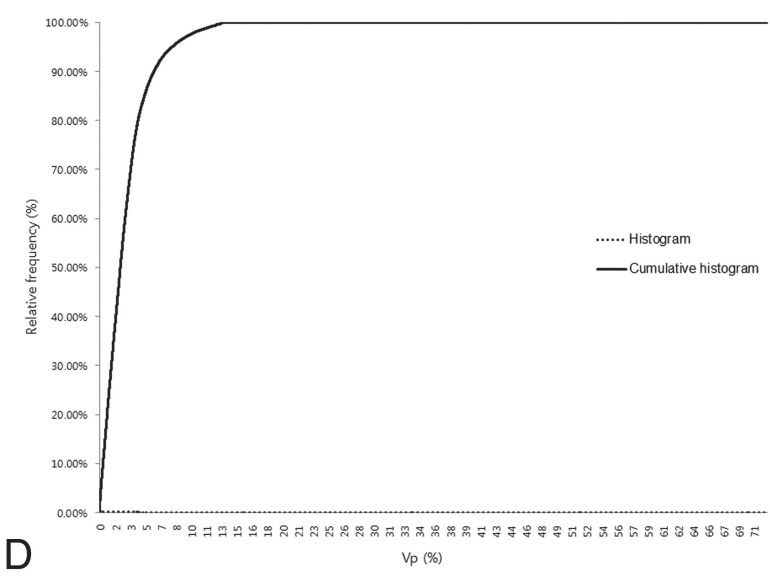

FIG 2. ROIs manually defined on axial T2WI coregistered with parametric maps in a 61-year-old man with a grade III glioma (B) and the histogram and cumulative histogram from the entire-tumor volume $(B-D)$ are presented.

lated in each tumor and parameter. Receiver operating characteristic (ROC) curves were generated to determine the optimum cutoff percentile for distinguishing high-grade from low-grade gliomas and differentiating grade III and IV tumors. The area under the curve (AUC), obtained from ROC analysis according to a specific percentile from C50 to C100, was analyzed. The percentiles with the highest AUC and the cutoff values with the best diagnostic performances (highest sensitivity and specificity) were presented in each parameter. The parameters with the best percentile values were compared by using a multivariable stepwise logistic regression model to determine the best predictors for differentiating high- and low-grade gliomas. ${ }^{27}$

\section{RESULTS}

Mean $K^{\text {trans }}, \mathrm{Ve}$, and $\mathrm{Vp}$ values are presented according to the tumor grade in Table 1 . The pharmacokinetic parameters increased with increasing glioma grade. There were significant differences among the grades for all parameters $(P<.01)$.

\section{Histogram Analysis}

The histograms and cumulative histograms from the entire-tumor volume showed that low-grade gliomas presented a higher relative frequency at low parametric values and a lower relative frequency at high parametric values compared with high-grade gliomas in all parameters. These characteristics resulted in substantial divergence according to grades in the cumulative histogram (Fig 4).

\section{Optimal Cutoff Values of $K^{\text {trans }}$, Ve, and Vp for the Differentiation of High- and Low-Grade Gliomas}

Between the C50 (median value) and C99 values from the cumulative $K^{\text {trans }}, \mathrm{Ve}$, and Vp histograms, the $K^{\text {trans }}$ of C98, Ve of C90, and Vp of C84 exhibited the highest AUCs-0.912, 0.939, and 0.769 - for differentiating high- from low-grade gliomas.

We applied each cutoff value of $K^{\text {trans }}, \mathrm{Vp}$, and $\mathrm{Ve}$ for the differentiation of high- and low-grade gliomas, so that tumors with values greater than or equal to the cutoff values were regarded as high-grade gliomas. In terms of $K^{\text {trans }}$, the cutoff value of C98 was 0.277 minute $^{-1}$ and showed a sensitivity of $76.19 \%$ (16 of 21 high-grade gliomas) and a specificity of $100 \%$ (7 of 7 lowgrade gliomas). The Ve cutoff value at C90 was $19.70 \%$ and had a sensitivity and a specificity of $85.7 \%$ ( 18 of 21 high-grade gliomas) and $100 \%$ (7 of 7 low-grade gliomas), respectively. We found that the Vp cutoff value at C84 was $11.71 \%$ and showed a sensitivity of $61.9 \%$ (13 of 21 high-grade gliomas) and a specificity of $100 \%$ (7 of 7 low-grade gliomas) (Table 2).

\section{Optimal Cutoff Values of $K^{\text {trans }}, V e$, and $V p$ for Differentiation of Grade II and III Gliomas}

Between the C50 and C99 values from the cumulative $K^{\text {trans }}, \mathrm{Vp}$, and Ve histograms, only Ve showed a significant parameter $(P<$ $.05)$ for differentiating grade III from grade II gliomas and the Ve C90 exhibited the highest AUCs: 0.839 . The Ve C90 cutoff value was $19.7 \%$ with a sensitivity of $62.5 \%$ ( 5 of 8 grade III gliomas) 


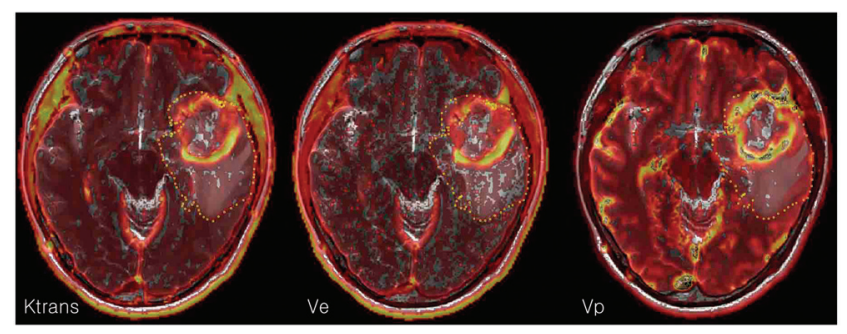

A

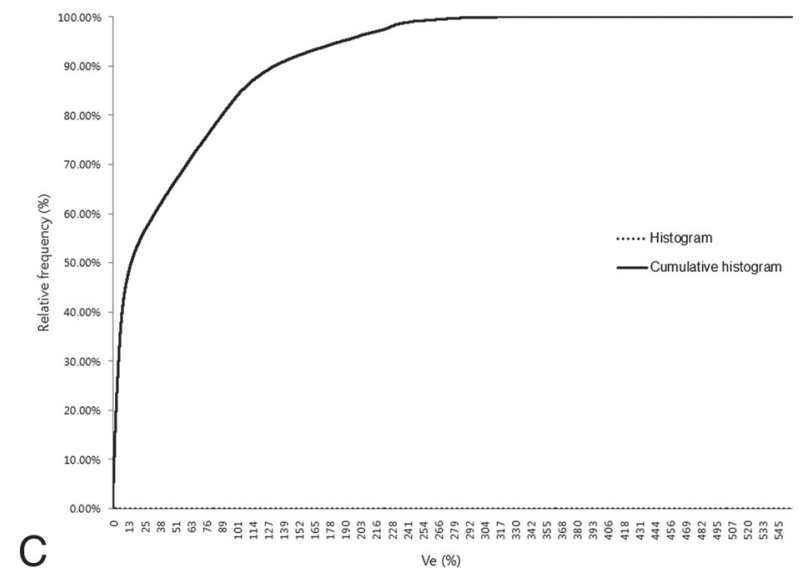

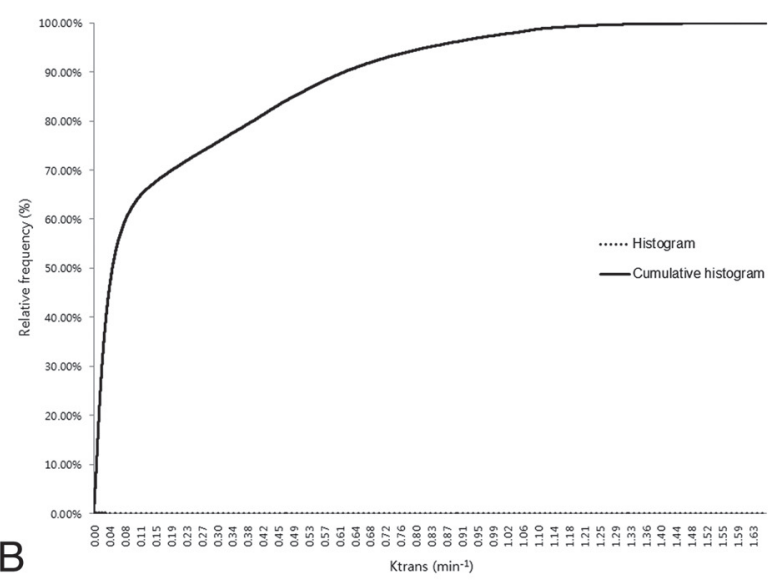

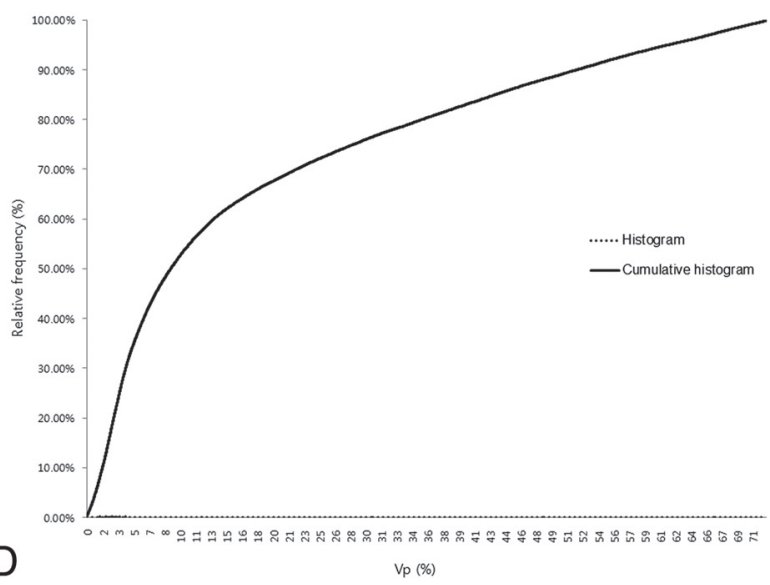

FIG 3. ROIs manually defined on axial T2WI coregistered with parametric maps in a 37-year-old woman with a grade IV glioma (C) and the histogram and cumulative histogram from the entire-tumor volume $(B-D)$ are presented.

Table 1: The pharmacokinetic parameters in each grade of glioma $^{a}$

\begin{tabular}{lcccc}
\hline & $\begin{array}{c}\text { Grade II }^{\mathrm{b}} / \\
\text { Low-Grade }^{\mathrm{c}}\end{array}$ & Grade III $^{\mathrm{b}}$ & Grade IV $^{\mathrm{b}}$ & High-Grade $^{\mathrm{c}}$ \\
\hline$K^{\text {trans d }}$ & $0.027 \pm 0.041$ & $0.057 \pm 0.091$ & $0.158 \pm 0.226$ & $0.122 \pm 0.195$ \\
$\mathrm{Ve}^{\mathrm{e}}$ & $5.809 \pm 10.381$ & $15.469 \pm 26.165$ & $45.103 \pm 63.888$ & $35.059 \pm 55.920$ \\
$\mathrm{Vp}^{\mathrm{e}}$ & $3.858 \pm 3.958$ & $5.735 \pm 5.772$ & $10.624 \pm 12.396$ & $8.826 \pm 10.723$ \\
\hline
\end{tabular}

all data are means.

${ }^{b}$ There were statistically significant differences among grades $(P<.01)$.

' There were statistically significant differences between low- and high-grade $(P<$ .01).

${ }^{\mathrm{d}}$ Data for $K^{\text {trans }}$ are in units of minute ${ }^{-1}$

${ }^{\mathrm{e}}$ Data for $V e$ and $V p$ are percentages.

and a specificity of $100 \%$ (7 of 7 grade III gliomas). We applied each cutoff so that tumors with values equal to or greater than the cutoff values were regarded as grade III gliomas.

\section{Optimal Cutoff Values of $\mathrm{K}^{\text {trans }}, \mathrm{Ve}$, and $\mathrm{VP}$ for the Differentiation of Grade III and IV Gliomas}

Between the C50 and C99 values from the cumulative $K^{\text {trans }}$, Vp, and Ve histograms, the $K^{\text {trans }}$ C97, Ve C66, and Vp C92 exhibited the highest AUCs for differentiating grade IV from grade III gliomas: $0.971,0.885$, and 0.798 , respectively. The $K^{\text {trans }} \mathrm{C} 97$ cutoff value was 0.412 minute $^{-1}$ with a sensitivity of $92.31 \%$ (12 of 13 grade IV gliomas) and a specificity of $100 \%$ (8 of 8 grade III gliomas). The Ve C66 cutoff value was $8.37 \%$ with a sensitivity of $92.31 \%$ (12 of 13 grade IV gliomas) and a specificity of $75 \%$ ( 6 of 8 grade III gliomas). We found that the Vp
C92 cutoff value was $19.33 \%$ and showed a sensitivity of $76.92 \%$ (10 of 13 grade IV gliomas) and a specificity of $87.50 \%$ (7 of 8 grade III gliomas). We applied each cutoff so that tumors with values equal to or greater than the cutoff values were regarded as grade IV gliomas.

\section{Multivariable Stepwise Logistic Regression Analysis}

The AUCs corresponding to profiles with the highest diagnostic accuracy showed significant differences between low- and highgrade gliomas $(P<.05)$. However, multivariable stepwise logistic regression analysis showed that the $K^{\text {trans }} \mathrm{C} 98$ value was the most significant variable, with a parameter estimate of 16.64 and a standard error of $8.17(P<.05) . K^{\text {trans }} \mathrm{C} 97$ was the most significant variable with a parameter estimate of 17.96 and a standard error of 8.38 in differentiating grade IV from grade III gliomas, but there was no significant variable in differentiating grade III from grade II gliomas $(P<.05)$.

\section{DISCUSSION}

Histogram analysis of pharmacokinetic parameters including $K^{\text {trans }}, \mathrm{Ve}$, and $\mathrm{Vp}$ obtained from the entire-tumor volume based on T1-weighted DCE perfusion MR imaging was useful for glioma grading. The best percentiles for differentiating high- and low-grade gliomas, in terms of the highest area under the curve of the receiver operating characteristic curves, were $K^{\text {trans }}$ C98, Ve 

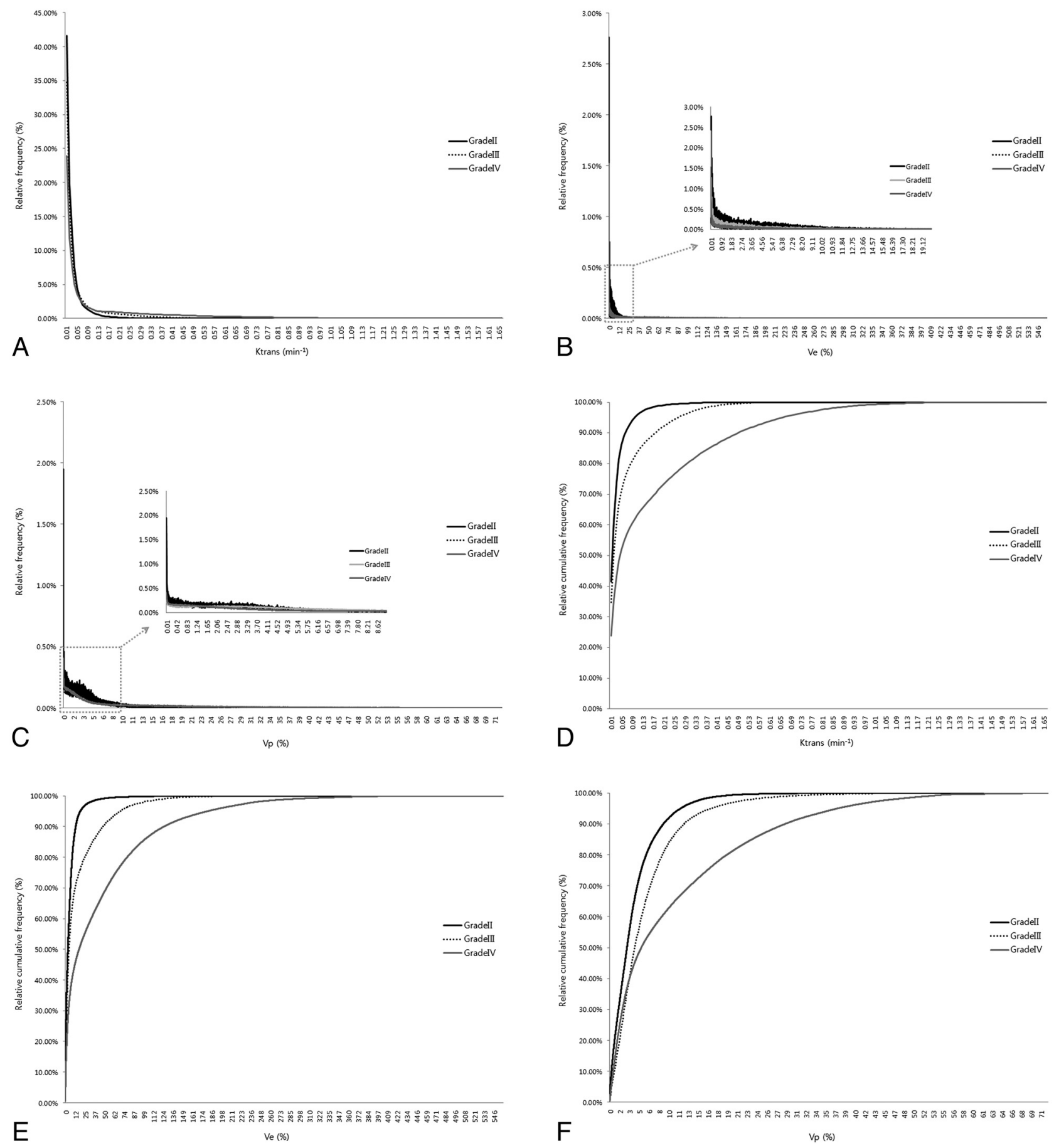

FIG 4. A histogram and cumulative histogram were derived from the total parametric values of all masses $(n=28)$ according to each grade. $A-F$, The histogram and cumulative histogram show that lower grade gliomas present a higher relative frequency at low parametric values and a lower relative frequency at high parametric values compared with higher grade gliomas for all of the parameters. $C$ and $F$, However, in the $V p$ histogram, the relative frequency of grade IV gliomas is higher than that of grade III gliomas at a number of low values.

C90, and Vp C84. $K^{\text {trans }}$ C98 was the most significant parameter for distinguishing high- from low-grade gliomas.

A number of studies on glioma grading by using pharmacokinetic parameters from DCE MR imaging-in particular, $K^{\text {trans }}$, $\mathrm{Ve}$, and $\mathrm{Vp}$ - have been published. ${ }^{8,10-13,15,18,28}$ Using ROC curve analyses, Jia et $\mathrm{al}^{8}$ showed that $K^{\text {trans }}$ and Ve were significantly lower in low-grade gliomas than in high-grade gliomas, with cutoff values of $K^{\text {trans }}$ and Ve of 0.035 minutes $^{-1}$ and 0.13 , respectively. Awasthi et al ${ }^{10}$ presented a discriminant function for differentiating low- and high-grade gliomas and a correlation with immunohistochemical markers by using relative CBV, elimination rate of contrast agent or Kep, and Ve derived from DCE MR imaging. Zhang et $\mathrm{al}^{29}$ concluded that $K^{\text {trans }}$ was the most sensitive and specific parameter among the 5 available parameters ( $K^{\text {trans }}$, Kep, rate constant of contrast agent reflux to plasma or $\mathrm{Kel}, \mathrm{Ve}$, and $\mathrm{Vp}$ ) in distinguishing high-grade from low-grade gliomas on the basis of DCE MR imaging. These studies calculated parameters in portions of the entire-tumor volumes. The ROIs 
Table 2: ROC results of the percentiles with the best AUC for glioma grading

\begin{tabular}{|c|c|c|c|c|c|}
\hline Parameter & $A \cup C^{a}$ & $\begin{array}{c}\text { Sensitivity } \\
(\%)^{\mathrm{b}}\end{array}$ & $\begin{array}{c}\text { Specificity } \\
(\%)^{\mathrm{b}}\end{array}$ & $\begin{array}{l}\text { Cutoff } \\
\text { Value }^{c}\end{array}$ & $\begin{array}{c}P \\
\text { Value }\end{array}$ \\
\hline \multicolumn{6}{|l|}{$K^{\text {trans }}$} \\
\hline C98 & $0.912(0.742-0.985)$ & 76.19 (16/21) & $100(7 / 7)$ & 0.277 & $<.01$ \\
\hline \multicolumn{6}{|l|}{$\mathrm{Ve}$} \\
\hline C90 & 0.939 (0.779-0.994) & 85.71 (18/21) & $100(7 / 7)$ & 19.70 & $<.01$ \\
\hline \multicolumn{6}{|l|}{$V p$} \\
\hline C84 & $0.769(0.572-0.906)$ & $61.9(13 / 21)$ & $100(7 / 7)$ & 11.71 & $<.01$ \\
\hline
\end{tabular}

${ }^{a}$ Data in parentheses are $95 \%$ confidence intervals.

${ }^{\mathrm{b}}$ Sensitivity and specificity for identifying high-grade tumors. Data in parentheses are the numbers used to calculate the percentages.

${ }^{c}$ Data for $K^{\text {trans }}$ are in units of minute ${ }^{-1}$, and data for $V e$ and $V p$ are percentages.

included smaller portions of the tumor margins on each section and were mostly used to show the highest parametric values. ${ }^{8,10,29}$ In comparison, we collected and analyzed whole-pixel values from the entire-tumor volume. In addition, we evaluated the parameters on the basis of T2WI or FLAIR, whereas previous studies used contrast- enhanced T1WI. T2-weighted high-signal-intensity lesions, excluding definite cystic or necrotic regions around gliomas, were generally considered to be tumor-involved lesions. We believe that the methods used in the present study could reflect whole-tumor characteristics.

The previous studies presented the parametric values with cutoff values to differentiate low- and high-grade gliomas. The presented values in the previous studies were not consistent with one another. The inconsistency in parametric values may be caused by different MR imaging protocols, pharmacokinetic models, or perfusion-analysis software. However, histogram analysis from the entire-tumor volume could be an alternative method for grading gliomas. The percentiles, which were generated from relative rankings based on the analyzed parametric values from the analysis, directly apply to other clinical or research settings.

Baseline T1 was fixed at $1000 \mathrm{~ms}$ in this study, whereas an estimate for the baseline $\mathrm{T} 1$ has been generally derived from a fitting process by using the precontrast $\mathrm{T} 1$ field-echo signal at multiple flip angles. ${ }^{20,30,31}$ The estimation of the baseline $\mathrm{T} 1$ is very sensitive to noise that is present in multiple flip angle and DCE images, and this sensitivity results in the broadening of the baseline $\mathrm{T} 1$. The fixed baseline $\mathrm{T} 1$ can contribute to obtaining more consistent results and can protect dynamic data from incorrect scale factors or patient movements during DCE data collection. In addition, the effort to estimate the actual baseline T1 could be reduced. If one assumed that the purpose of the DCE analysis is to determine the relative vascular change, the analysis by using the fixed baseline $\mathrm{T} 1$ may be sufficient to draw correct conclusions, even though the fixed baseline $\mathrm{T} 1$ is not physiologic. ${ }^{22}$ However, these characteristics may result in Ve values of $>100 \%$ in our study.

Although there were a number of methodologic differences, the results from this study were in agreement with those in previous studies. The parametric values increased as the grades increased, and $K^{\text {trans }}$ was the most significant parameter in glioma grading.

This study had a number of limitations. A retrospective analysis was performed, and a small number of patients were enrolled. In particular, only 7 cases of low-grade glioma were included because of their low incidence. Second, although the determination of the arterial input function was performed in terms of the best optical shape and in relation to the concentration-time curve of the tumor, it might be insufficient for objective and reproducible selection. Third, the drawing of the regions of interest was performed manually on T2WI or FLAIR images. Because infiltrative tumors show particularly poor margins, differentiating the tumor from the surrounding normal brain tissue may not be easy, and this difficulty may result in low reproducibility. However, the volume of data from the entire-tumor volume was high enough that small erroneous data were not thought to cause severe bias. Fourth, we assumed that significant differences in parameters among the glioma grades would be identified in higher percentiles over the range C50 to C100. This assumption was based on the characteristic vascular system of gliomas (ie, higher grade gliomas have more permeability and abundant vascularity). Although the AUC curves also supported the validity of the assumption, the analysis was not completely free from limitations. Fifth, multicolinearity was detected in the multivariable stepwise logistic regression analysis because the variables were highly correlated with one another. The percentiles were extracted from one parameter, and the parameters were originally dependent on one another. Sixth, fixed-baseline $\mathrm{T} 1$ was used for the calculation of contrast concentration in tissue, even though it was not physiologic. Finally, the histogram analysis of the data from the entire-tumor volume could present entire profiles of glioma grades on the basis of the calculated parameters, but this may not be practical in terms of time effectiveness for application to real clinical settings.

\section{CONCLUSIONS}

Histogram analysis of the pharmacokinetic parameters $K^{\text {trans }}$, Ve, and $\mathrm{Vp}$ obtained from the entire-tumor volume data can be useful for grading gliomas. $K^{\text {trans }} \mathrm{C} 98$ was the most significant parameter. We expect our results to contribute to perfusion analyses in diverse clinical and research settings.

Disclosures: Seung Hong Choi-RELATED: Grant: This study was supported by a grant from the National R\&D Program for Cancer Control, Ministry of Health and Welfare, Republic of Korea (1120300); the Korea Healthcare Technology R\&D Projects, Ministry for Health, Welfare and Family Affairs (A112028 and HI13C0015); and the Research Center Program of the Institute for Basic Science in Korea. * Money paid to the institution.

\section{REFERENCES}

1. Kargiotis O, Rao JS, Kyritsis AP. Mechanisms of angiogenesis in gliomas. J Neurooncol 2006;78:281-93

2. Folkman J. What is the evidence that tumors are angiogenesis dependent? J Natl Cancer Inst 1990;82:4-6

3. Folkman J. The role of angiogenesis in tumor growth. Semin Cancer Biol 1992;3:65-71

4. Wesseling P, Ruiter DJ, Burger PC. Angiogenesis in brain tumors; pathobiological and clinical aspects. J Neurooncol 1997;32:253-65

5. Less JR, Skalak TC, Sevick EM, et al. Microvascular architecture in a mammary carcinoma: branching patterns and vessel dimensions. Cancer Res 1991;51:265-73

6. Wesseling P, van der Laak JA, de Leeuw H, et al. Quantitative immunohistological analysis of the microvasculature in untreated human glioblastoma multiforme: computer-assisted image analysis of whole-tumor sections. J Neurosurg 1994;81:902-09

7. Tate MC, Aghi MK. Biology of angiogenesis and invasion in glioma. Neurotherapeutics 2009;6:447-57

8. Jia Z, Geng D, Xie T, et al. Quantitative analysis of neovascular permeability in glioma by dynamic contrast-enhanced MR imaging. J Clin Neurosci 2012;19:820-23

AJNR Am J Neuroradiol 35:1103-10 Jun 2014 www.ajnr.org

1109 
9. Hakyemez B, Erdogan C, Ercan I, et al. High-grade and low-grade gliomas: differentiation by using perfusion MR imaging. Clin $\mathrm{Ra}-$ diol 2005; 60:493-502

10. Awasthi R, Rathore RK, Soni P, et al. Discriminant analysis to classify glioma grading using dynamic contrast-enhanced MRI and immunohistochemical markers. Neuroradiology 2012;54:205-13

11. Provenzale JM, York G, Moya MG, et al. Correlation of relative permeability and relative cerebral blood volume in high-grade cerebral neoplasms. AJR Am J Roentgenol 2006;187:1036-42

12. Zhang $\mathrm{Y}$, Wang J, Wang $\mathrm{X}$, et al. Feasibility study of exploring a $\mathrm{T}$ (1)-weighted dynamic contrast-enhanced $M R$ approach for brain perfusion imaging. J Magn Reson Imaging 2012;35:1322-31

13. Harrer JU, Parker GJ, Haroon HA, et al. Comparative study of methods for determining vascular permeability and blood volume in human gliomas. J Magn Reson Imaging 2004;20:748-57

14. Lüdemann L, Grieger W, Wurm R, et al. Quantitative measurement of leakage volume and permeability in gliomas, meningiomas and brain metastases with dynamic contrast-enhanced MRI. Magn Reson Imaging 2005;23:833-41

15. Mills SJ, Patankar TA, Haroon HA, et al. Do cerebral blood volume and contrast transfer coefficient predict prognosis in human glioma? AJNR Am J Neuroradiol 2006;27:853-58

16. Aref M, Chaudhari AR, Bailey KL, et al. Comparison of tumor histology to dynamic contrast enhanced magnetic resonance imagingbased physiological estimates. Magn Reson Imaging 2008;26: 1279-93

17. Patankar TF, Haroon HA, Mills SJ, et al. Is volume transfer coefficient $\left(\mathrm{K}^{\text {trans }}\right)$ related to histologic grade in human gliomas? AJNR Am J Neuroradiol 2005;26:2455-65

18. Roberts HC, Roberts TP, Brasch RC, et al. Quantitative measurement of microvascular permeability in human brain tumors achieved using dynamic contrast-enhanced MR imaging: correlation with histologic grade. AJNR Am J Neuroradiol 2000;21:891-99

19. Tofts PS, Brix G, Buckley DL, et al. Estimating kinetic parameters from dynamic contrast-enhanced T(1)-weighted MRI of a diffusable tracer: standardized quantities and symbols. J Magn Reson Imaging 1999;10:223-32

20. Haroon HA, Buckley DL, Patankar TA, et al. A comparison of Ktrans measurements obtained with conventional and first pass pharma- cokinetic models in human gliomas. J Magn Reson Imaging 2004;19:527-36

21. Tofts PS, Kermode AG. Measurement of the blood-brain barrier permeability and leakage space using dynamic MR imaging. 1. Fundamental concepts. Magn Reson Med 1991;17:357-67

22. Haacke EM, Filleti CL, Gattu R, et al. New algorithm for quantifying vascular changes in dynamic contrast-enhanced MRI independent of absolute T1 values. Magn Reson Med 2007;58:463-72

23. Sundar H, Shen D, Biros G, et al. Robust computation of mutual information using spatially adaptive meshes. Med Image Comput Comput Assist Interv 2007;10(pt 1):950-58

24. Pluim JP, Maintz JB, Viergever MA. Mutual-information-based registration of medical images: a survey. IEEE Trans Med Imaging 2003;22:986-1004

25. Bjornerud A. The ICE software package: direct co-registration of anatomical and functional datasets using DICOM image geometry information. Proc Hum Brain Mapping New York, New York, June 19-22, 2003;19

26. Kang Y, Choi SH, Kim YJ, et al. Gliomas: histogram analysis of apparent diffusion coefficient maps with standard- or high-b-value diffusion-weighted MR imaging-correlation with tumor grade. Radiology 2011;261:882-90

27. Hauck WW, Miike R. A proposal for examining and reporting stepwise regressions. Stat Med 1991;10:711-15

28. Mills SJ, Soh C, O'Connor JP, et al. Enhancing fraction in glioma and its relationship to the tumoral vascular microenvironment: a dynamic contrast-enhanced MR imaging study. AJNR Am J Neuroradiol 2010;31:726-31

29. Zhang N, Zhang L, Qiu B, et al. Correlation of volume transfer coefficient Ktrans with histopathologic grades of gliomas. J Magn Reson Imaging 2012;36:355-63

30. Li KL, Zhu XP, Waterton J, et al. Improved 3D quantitative mapping of blood volume and endothelial permeability in brain tumors. $J$ Magn Reson Imaging 2000;12:347-57

31. Zhu XP, Li KL, Kamaly-Asl ID, et al. Quantification of endothelial permeability, leakage space, and blood volume in brain tumors using combined $\mathrm{T} 1$ and $\mathrm{T} 2^{\star}$ contrast-enhanced dynamic MR imaging. J Magn Reson Imaging 2000;11:575-85 